\title{
Editorials
}

\section{The future of academic anesthesia departments in Canada}

David R. Bevan MB MRCP FRCA FRCPC

I N 1945, Dr. Wesley Bourne was appointed to chair the Department of Anesthesia at McGill University, the first academic department of anesthesia in Canada. ${ }^{1}$ Over the next three decades academic anesthesia departments were established in all 16 Faculties of Medicine in Canada. Deans of Medicine were convinced that the specialty had a sufficient body of unique knowledge that further development of the discipline of anesthesia could be entrusted to University Departments. For 50 years academic anesthesia prospered: training programs developed that were envied by other specialties and were enhanced by the standards espoused by examiners of the Royal College of Physician and Surgeons of Canada and the Corporation Professionnelle des Médecins du Québec. But now, academic anesthesia is in trouble. In the United Kingdom, the Royal College of Anaesthetists has recently published a very depressing document "A National Strategy for Academic Anaesthesia" in response to the "severe crisis in academic anaesthesia." ${ }^{2}$ In the United States, there is concern over the declining proportion of publications by American authors in anesthesia journals. ${ }^{3}$

In Canada, our educational role means that our position is assured. University Departments of Anaesthesia in the United Kingdom envy the responsibility that Canadian Departments are given for both Undergraduate and Postgraduate education. United Kingdom Departments exist primarily as research centres without teaching responsibilities. Their funding is threatened by the "Research Assessment Exercise" that allocates resources for Higher Education according to the success of a Department or University in attracting independent grants and publishing in high impact journals. Only a few of the 24 academic anes- thesia departments (26 in 1995) are flourishing: some are headed by non-anesthesiologists and three chairs are vacant.

In Canada, anesthesia training has been characterized by innovations in teaching, particularly by the use of simulation, and in the examination process itself. Research, largely restricted to the process of anesthesia, has ensured that future developments in anesthesia pharmacology, monitoring technology (with the exception of a need for a device to measure anesthesia depth) and anesthesia practice will be small. These successes are evident in the decrease in anesthesia related mortality. ${ }^{4}$ Although the extent of the decrease may be debated, it has impressed the Canadian Medical Protective Association to decrease anesthesia membership fees. Anesthesia, led by the academic departments and its national society, the Canadian Anesthesiologists' Society (CAS), has put its own house in order and has evolved into a recognized specialty.

Despite these successes, the specialty has failed to influence the direction of medical practice in the $20^{\text {th }}$ century. Anesthesia has not been involved with the front-runners of research - molecular medicine, genomics and outcomes research. Anesthesia, in North America, plays a minimal role in Critical Care Medicine, making it even more important to continue to lead in the field of Pain Management and Research. More importantly, Canada has performed as badly or worse than most countries in ensuring a sufficient supply of specialist anesthesiologists to maintain current service loads. We are inadequately positioned to meet the demand of the baby-boomer population for increased access to health care in the next decade. ${ }^{5}$

From the Department of Anesthesia, University of Toronto, Toronto, Ontario, Canada.

Address correspondence to: Dr. David R. Bevan, Department of Anesthesia, Room 126, FitzGerald Building, 150 College Street,

Toronto, Ontario M5S 3E2, Canada. E-mail: david.bevan@utoronto.ca 
The role of the academic department

The role of academic departments has not changed. They act to store existing knowledge, to create new knowledge and, in Canada, to train future anesthesiologists. Until now, such teaching and research have been introspective - directed internally to the specialty of anesthesia. In future, the skills learned and knowledge gained by our specialty should be focused more widely - to the whole faculties of medicine, nursing and allied health.

\section{Teaching}

The traditional doctor-patient relationship based on the one-on-one presence of the anesthesiologist to the surgical patient has provided an environment where the anesthesia student, undergraduate and postgraduate, receives maximal stimulation. Such teaching is augmented in all university departments by rounds, academic days, specialist courses and Continuing Medical Education on every conceivable topic. Surprisingly, despite the long history of teaching excellence, only a few anesthesiologists, such as the late Dr. John Bradley, have taken a leadership role in medical education outside anesthesia.

There are new opportunities for us to grasp. A revolution has taken place in medical education - simulation. Most anesthesia departments now incorporate simulation into their programs. Anesthesiologists recognized the potential of simulation earlier than most other specialists not only for technical procedures such as tracheal intubation and vascular access but also in improving patient safety. Exposure to crisis management in the simulated environment demonstrated the importance of personal interactions in clinical situations. The operating room is analogous to the airplane cockpit and similar behaviour is necessary to avoid and manage unexpected events. ${ }^{6}$ Other groups, including nurses, medical students, allied health professionals, and surgeons recognize the importance of initial technical exposure on a mannequin to learn clinical skills before approaching the patient. Anesthesiologists should be sharing their skills throughout medicine. Not only do they have the experience, but they have developed the critical evaluative processes and, in the whole-body simulator, have brought a new level of understanding to team care. All faculties of medicine require access to a sophisticated simulation environment. Anesthesiologists are well placed to lead the field and exert a presence across all medical practice. Patients, quite naturally, are reluctant guinea-pigs for trainee medical personnel. Anesthesiologists must take the lead in the revolution.

\section{Research}

Research in the first 50 years of academic anesthesia in Canada was largely concerned, in partnership with the pharmaceutical industry, with the development of safe and dependable drugs. Further major modifications to our armamentarium of general and local anesthetic, neuromuscular blocking drugs, or sedatives are unlikely, although research will continue into analgesic drugs to reduce their toxicity and to improve their efficacy. Sophisticated basic science research will continue to elucidate the mechanism(s) of action and optimal drug delivery of general ${ }^{7}$ and local ${ }^{8}$ anesthetics in the hope that new, targeted drugs result. However, the huge cost in developing new compounds together with the small improvements will limit investment by pharmaceutical companies into anesthesia research.

Future pharmacological research is much more likely to be directed towards the identification of abnormal responders to existing compounds in collaboration with genetic investigators. Indeed, anesthesia was pivotal to the development of pharmacogenetics when Kalow identified abnormal cholinesterase enzymes in the metabolism of succinylcholine ${ }^{9}$ and in the recognition and management of malignant hyperthermia. ${ }^{10}$ Abnormal reactions to anesthetic agents will continue to be investigated for possible genetic linkage. On a wider scale, genetic variants and their influence on intraoperative bleeding and thrombosis ${ }^{11}$ may be important components of preoperative evaluation by the anesthesiologist.

Much of what we do in the perioperative period is in treating and preventing organ dysfunction. Of particular importance have been our attempts to improve cardiac outcomes by preoperative $\beta$-adrenergic blockade, calcium channel blockers, epidural anesthesia, etc. ${ }^{12}$ We know that the conduct of anesthesia influences patient outcome well after the end of surgery. Anesthesiologists are now involved in some of the most comprehensive outcome studies but these are difficult, time-consuming and require epidemiologists both within and outside anesthesia departments. Some traditional myths, such as the ubiquitous use of $\beta$-adrenergic blocking drugs in the cardiac patient, ${ }^{13}$ are being criticized. Interestingly, it may not be the treatment, but the identification of the right patient that is important. For example, the advantageous effect of $\beta$-blocking in acute cardiac syndromes may depend on the genetically determined type of $\beta$ adreno-receptor. ${ }^{14}$ Preoperative preparation and risk evaluation have been, and will remain the preserve of the anesthesiologist if we want it! 


\section{Anesthesia service provision}

In Canada, academic departments are responsible for the training of anesthesiologists. Approximately half the specialist anesthesiologists are employed in Academic Health Science Centres. The traditional Canadian anesthesia model, espoused by the CAS Guidelines, requires a single anesthesiologist for each anesthetized patient but there are insufficient anesthesia personnel to meet existing demands. Currently, academic and community hospitals have shortages of 10 to $15 \%$ in meeting this requirement. ${ }^{5}$ The traditional model has failed over the last decade because the training centres, academic hospitals, could not provide an alternative to meet the current and likely increasing demand for anesthesia. Worse, the difficulty in providing clinical anesthesia has caused the teaching hospital personnel to relinquish their academic roles to provide clinical service.

Additional personnel are necessary to meet current service needs for anesthesia. Also, academic anesthesiologists' time must be protected to ensure the continuing education of future specialists. There are few solutions: immigration of foreign trained physician specialists, re-training family practitioners, anesthesia nurses and specially trained anesthesia assistants. In Canada, there is little enthusiasm for overseas physician recruitment or the training of an alternative group of anesthesia suppliers, anesthesia nurses. There is growing support for the training of health professionals to assist the anesthesiologist, but while the patient always remains under the care of the specialist physician. The concept of an anesthesiologist-led anesthesia care team incorporating preoperative preparation, intraoperative care and postoperative pain management is appealing. The functions of the anesthesiologist extend beyond the operating room. Responsibility for acute postoperative pain control is particularly important to ensure that the care is provided by those most familiar with the techniques used in its function. Any change will require careful evaluation of the necessary training programs and continuous assessment of their effectiveness and safety. The academic departments must be involved.

\section{Role of the Royal College of Physicians and Surgeons of Canada}

Mention has already been made for the important role of the Royal College in determining the direction of the training of anesthesiologists. Its role has emphasized the achievement of the high level of clinical skills required in the operating room. The recent implementation of a new framework of core competencies for all specialists, "CanMEDS Roles" derived from "Canadian
Medical Education Directives for Specialists". The competencies include the roles of Medical Expert (central role), Communicator, Collaborator, Health Advocate, Scholar and Professional. ${ }^{15}$ Inevitably, such changes in emphasis will encourage the College to re-examine how the five years of postgraduate education in anesthesia need to be altered to meet these goals, and this will require leadership from the academic departments.

\section{A road map for the $21^{\text {st }}$ century}

As Yogi Berra once said: "When you arrive at a fork in the road, take it."

The future of anesthesia may take several directions. My sign-posts would include:

1) Become the leaders of medical education based on simulation

2) Focus research on relevant perioperative areas including:

a. Perioperative risk reduction

b. Genetic linkage

c. Blood conservation

3) Health care modelling - augmenting anesthesia supply to match demand.

In each of these areas academic anesthesia will be important in framing the questions, collecting and evaluating the data, and implementing change.

\section{Conclusion}

The first 50 years of academic anesthesia in Canada have been remarkably successful in producing highly skilled practitioners and organized departments that have provided excellent clinical care. There are two problems: the number of anesthesiologists is inadequate, and the contribution to the science of medicine has been too parochial. The next decade should be directed towards a team approach to anesthesia and to ensure that anesthesia teaching and research are wide in scope and direction so that anesthesia is seen to contribute to the practice of medicine.

\section{L'avenir des départe- ments universitaires d'anesthésie au Canada}

En 1945, le Dr Wesley Bourne a été nommé directeur du Département d'anesthésie à l'université McGill, le premier au Canada. ${ }^{1}$ Au cours des trois décennies 
suivantes, des départements universitaires d'anesthésie ont été créés dans les 16 facultés de médecine du pays. Les doyens de médecine étaient convaincus que la spécialité présentait un bagage suffisant de connaissances propres pour que les futures réalisations en anesthésie soient confiées à des départements universitaires. L'anesthésie universitaire a prospéré pendant 50 ans : des programmes de formation ont été élaborés, qui ont fait l'envie d'autres spécialités et qui ont été améliorés par les normes adoptées par les examinateurs du Collège royal des médecins et chirurgiens du Canada et la Corporation Professionnelle des Médecins du Québec. Mais maintenant, l'anesthésie universitaire est en difficulté. Au Royaume-Uni, le Royal College of Anaesthetists a récemment publié un document très déprimant, A National Strategy for Academic Anaesthesia (Une stratégie nationale pour l'anesthésie universitaire) en réponse au "severe crisis in academic anaesthesia» (sévère crises en anesthésie universitaire). ${ }^{2}$ Aux États-Unis, on est préoccupé par le déclin de la proportion de publications par les auteurs américains dans les revues d'anesthésie. ${ }^{3}$

Au Canada, notre rôle pédagogique fait en sorte que notre position est assurée. Les départements universitaires d'anesthésie du Royaume-Uni envient la responsabilité que les départements canadiens se sont donnée pour l'enseignement doctoral et postdoctoral. Les départements du Royaume-Uni existent principalement comme centres de recherche sans responsabilités d'enseignement. Leur financement est menacé par le «Research Assessment Exercise» qui alloue des ressources pour les études supérieures selon le succès d'un département ou d'une université à attirer des bourses indépendantes et à publier dans des revues à impact élevé. Seuls quelques-uns des 24 départements universitaires d'anesthésie (26 en 1995) sont florissants : certains sont dirigés par des nonanesthésiologistes et trois postes de directeur sont vacants.

Au Canada, la formation en anesthésie a été caractérisée par des innovations dans l'enseignement, en particulier par l'usage de la simulation, et dans le processus d'examen lui-même. En recherche, grandement restreinte au processus de l'anesthésie, on s'est assuré que les futures avancées en pharmacologie de l'anesthésie, en technologie du monitorage (avec l'exception du besoin d'un appareil pour mesurer la profondeur de l'anesthésie) et en pratique de l'anesthésie soient limitées. Ces succès sont mis en évidence par la baisse de la mortalité reliée à l'anesthésie. ${ }^{4}$ On peut discuter de l'étendue de cette baisse, mais elle a incité l'Association canadienne de protection médicale à réduire les primes d'assurance en anes- thésie. Dirigée par les départements universitaires et sa société nationale, la Société canadienne des anesthésiologistes (SCA), l'anesthésie a mis de l'ordre dans ses affaires et est devenue une spécialité reconnue.

Malgré ces succès, la spécialité a failli dans sa tâche d'influencer la direction de la pratique médicale au $20^{\mathrm{c}}$ siècle. L'anesthésie n'a pas participé aux dossiers de l'heure - la médecine moléculaire, la génomique et la recherche sur les résultats. En Amérique du Nord, elle joue un rôle minimal en médecine intensiviste. Il est donc d'autant plus important de rester le chef de file dans le domaine de la recherche et du contrôle de la douleur. Qui plus est, le Canada a affiché une performance aussi mauvaise ou pire que la plupart des pays à s'assurer des effectifs suffisants d'anesthésiologistes spécialisés pour maintenir les charges de travail actuelles. Nous sommes mal placés pour répondre à la demande des baby-boomers d'augmenter l'accès aux soins de santé pour la prochaine décennie. ${ }^{5}$

\section{Le rôle du département universitaire}

Le rôle des départements universitaires n'a pas changé. Ils gardent les connaissances existantes, créent du nouveau savoir et, au Canada, forment les futurs anesthésiologistes. Jusqu'à présent, l'enseignement et la recherche ont été introspectifs - dirigés à l'interne vers la spécialité de l'anesthésie. À l'avenir, les techniques et les connaissances acquises par la spécialité devront s'ouvrir - vers toutes les facultés de médecine, de soins infirmiers et de services paramédicaux.

\section{L'enseignement}

La relation médecin-patient traditionnelle fondée sur la présence exclusive de l'anesthésiologiste auprès de l'opéré a créé un environnement où l'étudiant en anesthésie, de niveau prédoctoral ou postdoctoral, reçoit une stimulation maximale. Cet enseignement est augmenté dans tous les départements universitaires par des rotations, des journées d'études, des cours spécialisés et la formation médicale continue sur tout sujet imaginable. Curieusement, malgré la longue histoire d'excellence de l'enseignement, peu d'anesthésiologistes, comme feu le Dr John Bradley, ont tenu un rôle de premier plan en formation médicale à l'extérieur de l'anesthésie.

Il y a de nouvelles occasions à saisir pour nous. Une révolution s'est produite dans la formation médicale - la simulation. La majorité des départements d'anesthésie l'incluent maintenant dans leurs programmes. Les anesthésiologistes ont reconnu son potentiel plus tôt que beaucoup d'autres spécialistes, non seulement pour les interventions techniques comme l'intubation endotrachéale et l'accès vasculaire, mais aussi pour 
l'amélioration de la sécurité du patient. L'exposition à la gestion de crise dans un environnement simulé a démontré l'importance des interactions personnelles dans les situations cliniques. La salle d'opération est analogue à la cabine de pilotage d'un avion et exige des réactions semblables pour éviter et gérer des événements inattendus. ${ }^{6}$ D'autres groupes, dont les infirmières, les étudiants de médecine, les professionnels paramédicaux et les chirurgiens, reconnaissent l'importance du premier essai sur un mannequin pour apprendre des techniques cliniques avant de rencontrer le patient. Les anesthésiologistes devraient partager leurs compétences avec tous leurs collègues médecins. Non seulement ont-ils l'expérience, mais ils ont aussi développé des processus d'évaluation critique et, avec le simulateur du corps entier, ont apporté un nouveau degré de compréhension des soins en équipe. Toutes les facultés de médecine ont besoin d'un accès à un environnement de simulation complexe. Les anesthésiologistes sont bien placés pour diriger et participer à toute la pratique médicale. Les patients, plutôt naturellement, sont réticents à servir de cobayes pour le personnel médical stagiaire. Les anesthésiologistes doivent mener la révolution.

\section{La recherche}

La recherche des 50 premières années de l'anesthésie universitaire au Canada a largement porté sur le développement, en partenariat avec l'industrie pharmaceutique, de médicaments sûrs et fiables. D'autres modifications importantes à notre arsenal thérapeutique d'anesthésiques locaux et généraux, de curarisants ou de sédatifs sont peu probables, quoique la recherche va se poursuivre sur les analgésiques pour réduire leur toxicité et améliorer leur efficacité. La recherche raffinée en science fondamentale va continuer à élucider les mécanismes d'action et l'administration optimale des anesthésiques généraux ${ }^{7}$ et locaux ${ }^{8}$ en espérant que de nouveaux médicaments, ciblés, vont en résulter. Cependant, l'énorme coût rattaché à la recherche de nouveaux composés et des améliorations minimes vont limiter l'investissement des compagnies pharmaceutiques dans la recherche en anesthésie.

À l'avenir, la recherche pharmacologique, en collaboration avec la recherche en génétique, sera fort probablement dirigée vers l'identification des sujets qui réagissent anormalement aux composés existants. En effet, l'anesthésie a été le pivot du développement de la pharmacogénétique, avec Kalow qui détecte les enzymes anormaux de cholinestérase dans le métabolisme de la succinylcholine, ${ }^{9}$ et de la reconnaissance et du traitement de l'hyperthermie maligne. ${ }^{10}$ Les réactions anormales aux anesthésiques seront encore à l'étude pour de possibles liens génétiques. À une plus grande échelle, les variantes génétiques et leur influence sur les saignements peropératoires et la thrombose ${ }^{11}$ peuvent être des composantes importantes de l'évaluation préopératoire par l'anesthésiologiste.

Une grande part de notre travail périopératoire est la prévention et le traitement de la dysfonction organique. Nos tentatives pour améliorer les résultats cardiaques par un blocage $\beta$-adrénergique préopératoire, des inhibiteurs calciques, l'anesthésie péridurale etc. $^{12}$ ont été d'une particulière importance. Nous savons que la conduite de l'anesthésie influence l'état du patient bien après la fin de l'opération. Nous participons actuellement à quelques-unes des plus vastes études de résultats, mais ces études sont longues et difficiles et exigent des épidémiologistes à l'intérieur comme à l'extérieur des départements d'anesthésie. Quelques idées reçues, comme l'usage omniprésent des bloqueurs $\beta$-adrénergiques chez le patient cardiaque, ${ }^{13}$ sont critiquées. Ce qui est important, ce n'est peut-être pas le traitement, mais de savoir qui est le bon patient. Ainsi, l'effet bénéfique des $\beta$-bloquants dans le cas de syndromes cardiaques aigus peut dépendre du type d'adréno-récepteurs $\beta$ déterminés génétiquement. ${ }^{14} \mathrm{La}$ préparation préopératoire et l'évaluation du risque ont été, et demeureront réservées à l'anesthésiologiste si nous le voulons !

\section{La prestation de services d'anesthésie}

Au Canada, les départements universitaires sont responsables de la formation des anesthésiologistes. Environ la moitié des anesthésiologistes spécialisés travaillent dans des Centres des sciences de la santé. Le modèle traditionnel de l'anesthésie au Canada, proposé par le Guide de pratique de la SCA, exige un anesthésiologiste pour chaque patient anesthésié, mais le personnel d'anesthésie est insuffisant pour répondre aux demandes. Présentement, les hôpitaux universitaires et communautaires ont des pénuries de 10 à $15 \%$ par rapport aux besoins. ${ }^{5}$ Le modèle traditionnel a échoué au cours de la dernière décennie parce que les centres de formation, les hôpitaux universitaires, n'ont pu offrir d'autres solutions pour répondre à la demande actuelle, et probablement croissante, d'anesthésie. Pire encore, la pénurie en anesthésie clinique a forcé les hôpitaux universitaires à abandonner l'enseignement pour la clinique.

Il faut du personnel supplémentaire pour répondre aux besoins de service actuels en anesthésie. Il faut aussi protéger le temps des anesthésiologistes professeurs pour assurer la formation continue de futurs spécialistes. Il y a peu de solutions : l'immigration de médecins 
spécialistes formés à l'étranger, le perfectionnement des médecins de famille, les infirmières anesthésistes et les assistants en anesthésie spécialement formés. Au Canada, il y a peu d'enthousiasme pour le recrutement de médecins d'outre-mer ou la formation d'autres groupes d'anesthésie, les infirmières anesthésistes. Par contre, il y a un appui croissant à la formation de professionnels de la santé qui assisteraient l'anesthésiologiste, le patient demeurant constamment sous les soins du médecin spécialiste. Le concept d'une équipe de soins d'anesthésie dirigée par l'anesthésiologiste comprenant la préparation préopératoire, les soins peropératoires et le contrôle de la douleur postopératoire est attirant. Les fonctions de l'anesthésiologiste s'exercent au delà de la salle d'opération. La responsabilité du contrôle de la douleur postopératoire aiguë est particulièrement importante pour assurer des soins fournis par ceux qui connaissent le mieux les techniques utilisées dans ce but. Tout changement va demander une évaluation soigneuse des programmes de formation nécessaires et une évaluation continue de leur efficacité et de leur sécurité. Les départements universitaires doivent participer.

\section{Le rôle du Collège royal des médecins et chirur- giens du Canada}

Nous avons déjà parlé du rôle important du Collège royal dans la détermination des orientations de la formation des anesthésiologistes. Son rôle a été d'insister sur le haut niveau de compétence clinique nécessaire dans la salle d'opération. La récente introduction d'un nouveau cadre de compétences essentielles pour tous les spécialistes, «Rôles CanMEDS» vient du «Canadian Medical Education Directives for Specialists» ou "Cadre de compétences essentielles pour les médecins spécialisés canadiens». Les compétences incluent les rôles d'expert médical (rôle principal), de communicateur, de collaborateur, de promoteur de la santé, de gestionnaire, d'érudit et de professionnel. ${ }^{15}$ Inévitablement, de pareils changements de mise en valeur vont encourager le Collège à revoir comment les cinq ans d'études postdoctorales en anesthésie doivent être modifiées pour atteindre ces objectifs, ce qui va exiger de l'initiative des départements universitaires.

Une carte routière pour le $21 \mathrm{e}$ siècle

Comme l'a dit un jour Yogi Berra : "Sur une route, quand tu arrives à une fourche, prends-la.»

L'avenir de l'anesthésie peut emprunter différentes directions :

1) Devenir les chefs de file de la formation médicale fondée sur la simulation
2) Centrer la recherche sur les champs périopératoires dont :
a. La réduction du risque périopératoire
b. Les liens génétiques
c. La conservation du sang

3) Modéliser les soins de santé - en augmentant les services d'anesthésie pour répondre à la demande.

Dans chacun de ces domaines, l'anesthésie universitaire sera importante pour formuler les questions, recueillir et évaluer les données et appliquer les changements.

\section{Conclusion}

Les 50 premières années de l'anesthésie universitaire au Canada ont été remarquablement fructueuses, car elles ont donné des praticiens de grande compétence et organisé des départements qui ont fourni d'excellents soins cliniques. Deux problèmes existent toutefois: le nombre d'anesthésiologistes est insuffisant et la participation à la science médicale a été trop fermée. La prochaine décennie doit être dirigée vers une approche d'équipe de l'anesthésie et doit s'assurer que la formation et la recherche en anesthésie soient largement ouvertes et contribuent à la pratique de la médecine.

\section{References}

1 Bevan JC, Pacelli M. Wesley Bourn: The Quintessential Canadian Anaesthetist. McGill University Libraries, 1996.

2 Royal College of Anaesthetists. A National Strategy for Academic Anaesthesia (December 2005). URL available from: www.rcoa.ac.uk (accessed February 23, 2006).

3 Szokol JW, Murphy GS, Avram MJ, Nitsun M, Wynnychenko TM, Vender JS. Declining proportion of publications by American authors in major anesthesiology journals. Anesth Analg 2003; 96: 513-7.

4 Lienhart A, Auroy $\Upsilon$, Pequignot F, et al. Preliminary results from the SFAR-INSERM inquiry on anaesthesia-related deaths in France: mortality rates have fallen ten-fold over the past two decades (French). Bull Acad Natl Med 2004; 188: 1429-37.

5 Engen DA, Morewood GH, Ghazar NJ, Ashbury T, VanDenKerkhof EG, Wang L. A demand-based assessment of the Canadian anesthesia workforce - 2002 through 2007. Can J Anesth 2005; 52: 18-25.

6 Gaba DM, Fish KJ, Howard SK. Crisis Management in Anesthesiology. New York: Churchill Livingstone; 1994.

7 Hemmings HC Jr, Akabas MH, Goldstein PA, Trudell $J R$, Orser BA, Harrison NL. Emerging molecular 
mechanisms of general anesthetic action. Trends Pharmacol Sci 2005; 26: 503-10.

8 Chan VW, Nova H, Abbas S, McCartney CJL, Perlas $A, X u d a$ Q. Ultrasound examination and localization of the sciatic nerve: a volunteer study. Anesthesiology 2006; 104: 309-14.

9 Kalow W, Staron N. On distribution and inheritance of atypical forms of human serum cholinesterase, as indicated by dibucaine number. Can J Biochem Physiol 1957; 35: 1305-20.

10 Monnier N, Kozak-Ribben G, Krivosic-Horber R, et al. Correlations between genotype and pharmacological, histological, functional, and clinical phenotypes in malignant hyperthermia susceptibility. Hum Mutat 2005; 26: 413-25.

11 Donahue BS, Gailani D, Higgins MS, Drinkwater DC, George $A L J r$. Factor V Leiden protects against blood loss and transfusion after cardiac surgery. Circulation 2003; 107: 1003-8.

12 Lindenaner PK, Pekow P, Wang K, Mamidi DK, Gutierrez B, Benjamin EM. Perioperative beta-blocker therapy and mortality after major noncardiac surgery. N Engl J Med 2005; 353: 349-61.

13 Devereaux PJ, Beattie WS, Choi PT, et al. How strong is the evidence for the use of perioperative beta blockers in non-cardiac surgery? Systematic review and meta-analysis of randomised controlled trials. BMJ 2005; 331: 313-21.

14 Lanfear DE, Jones PG, Marsh S, Cresci S, McLeod HL, Spertus JA. $\beta_{2}$.Adrenergic receptor genotype and survival among patients receiving $\beta$-blocker therapy after an acute coronary syndrome. JAMA 2005; 294: 1526-33.

15 The CanMEDS Project Overview. Royal College of Physicians and Surgeons of Canada. URL available from: www.rcpsc.medical.org (accessed February 23, 2006). 